\title{
Mobile Support for Team-Based Field Surveys
}

\author{
Malcolm Hall, Philip Gray \\ Department of Computing Science, University of Glasgow, Glasgow, G12 8QQ, \\ United Kingdom \\ $\{$ mh, pdg\}@dcs.gla.ac.uk \\ http://www.dcs.gla.ac.uk
}

\begin{abstract}
This paper describes a study of the use of multimedia networked location-aware mobile computers to support team-based survey-oriented fieldwork. Existing systems do not provide fully integrated support for collaborative data capture and review, or access to distributed real time information on survey progress and status, all of which are crucial for the conduct and management of surveys often carried out under inflexible time constraints. We developed a mobile system to address these shortcomings and performed an evaluation in an archaeological field survey, supporting over twohundred data collection incidents over five days, and providing further insight into the field work data collection process.
\end{abstract}

\section{Introduction}

Mobile computers, wireless networking and positioning technologies are becoming increasingly suitable for outdoor work. However, determining how to use them effectively in team environments still remains a challenge. Work by Fagrell et al [1], and a number of commercial systems, demonstrates the value of mobile computers supporting teams of outdoor workers; particularly for collaboration and the coordination of activities. Team-based field surveys, such as archaeological field studies, are a promising application area for these technologies, since they involve intense collaboration over a distributed area. In team-based field surveys two essential requirements are positional awareness and team coordination. Mobile technologies can not only meet their current requirements, but potentially offer unique, previously unrealised benefits through the real time update of information on fieldwork progress supporting timely coordination of the team effort.

This paper describes our proposal of a multimedia oriented, location aware system to support team-based field studies. This system allows field workers to share their position and activity with other fieldworkers, as well as collecting photographs and data at their current location, and share this wirelessly with other fieldworkers. We present the results of an initial requirements capture involving professional archaeologists, and the resulting prototype system addressing their needs. Finally, we describe an evaluative field trial of the system and conclude with observations on the potential of this technology. 


\section{Supporting team-based field work}

Pascoe et al [2] defined four characteristics of the mobile field worker - "dynamic user configuration, limited attention capacity, high speed interaction and context dependency" and subsequently developed the Minimal Attention User (MAU) interface. Fieldnote [3] is their proposed system featuring a MAU interface which utilises a database to allow the collation of users' data. While innovative in terms of its interface, the Fieldnote system has no real-time synchronous support for teamwork. Other systems such as Renevier and Nigay's [4] 'MAGIC' system, and RAMSES [5] have similar shortcomings in that they focus on single user data collection and fail to support synchronous collaborative fieldwork.

The importance of team work in archaeology became apparent to us in our early design studies of archaeological work. We started by examining a typical field survey, part of the South East Melos Project ${ }^{1}$ whose aim was to locate evidence of early mines and Roman quarries. Through interviews and observations we were able to outline the areas where computer support would be beneficial and identified some key requirements.

In the typical archaeological field survey we studied, several small teams are each assigned to a particular sector of the survey area and responsible for recording and investigating all features of interest. Each team needs to be aware of its location in its assigned sector and should be able to create records, visual or otherwise, of its findings. Post-survey the data needs to be gathered and organised into an easily accessible format to facilitate further study.

To assist location awareness the Melos team used handheld GPS devices, a compass, an aerial photo and a map. It was noted that using the equipment together was a time-consuming and laborious process, further aggravated by the apparent incompatibility between the area map coordinate system provided and those supported by their GPS units. This suggests the possibility of support for automatically overlaying GPS information onto a geo-referenced digital map.

The creation and cataloguing of records was addressed by the Melos team using paper forms and a digital camera. This proved problematic as the task of physical record management was left to the user. In fact, it was often the case that paper forms were misplaced or lost by a surveyor during an expedition. Finally, management and data entry of gathered records was originally performed manually by project leaders in the Melos group through a lengthy and error-prone process.

\section{System design}

To support these collaborative activities during field work our system uses a clientserver architecture. The server is designed to be deployed on-site in order to coordinate data exchange among the client devices, and act as a central record repository. The client is designed to operate on several lightweight mobile devices, with limited processing abilities. In practice we expect our system to be used with a single server

\footnotetext{
${ }^{1}$ http://www.gla.ac.uk/archaeology/projects/melwww/agkhome.html
} 
and 5-10 client devices, one for each team, though provisions have been made for scalability.

The client is a Java application which provides a map viewer and record entry components. The map viewer (see left side figure 1) displays a geo-referenced map image and supports panning and zooming using a stylus or the directional button. The user's current location is displayed as a marker, and is broadcast to the other users, allowing all users' locations to be displayed. Similarly, markers are used to display the location at which records were created, and this information is distributed to all users. The map displays polygons marking the area each team has been allocated to survey, and also indicates the area covered. These features aid orientation and provide easily accessible insight on a team's progress. Other features enhancing usability include a distance measurement tool and the ability to re-position previously recorded observations on the map.
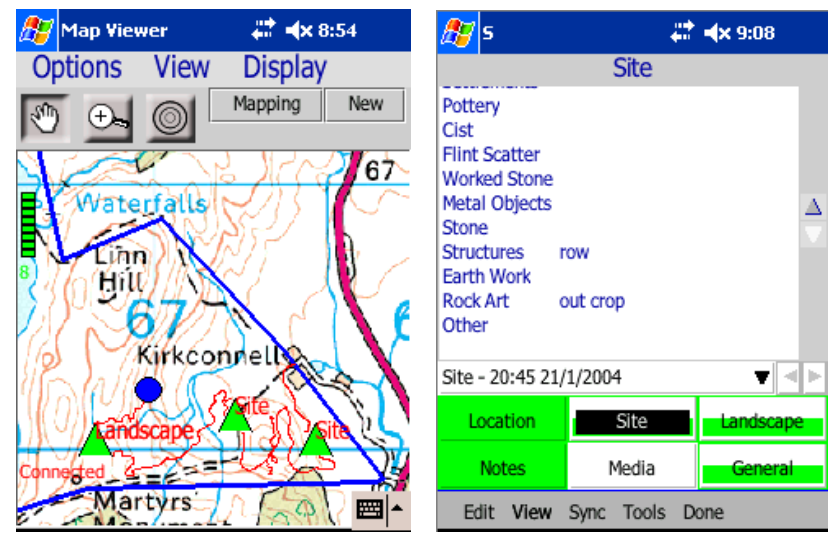

Fig. 1. The map and form interfaces to the client application

Record entry is handled via the form view (see right side figure 1). Each record entry is divided into sections which are accessible using the panel at the bottom. The buttons are colour-coded to convey each section's completion status.

The sections are as follows: a GPS section which is automatically filled when the record is created; a media section which displays the camera viewfinder and allows the attachment of photos and voice recordings; structured input sections for the records contents; and a section for general notes. The form structure is flexible as it is specified in XML and can be easily altered using a form builder application. The form engine ${ }^{2}$ is based on the Paraglide system [6] and utilises optimized input techniques, such as pick lists and auto-completion.

The server component of the system is implemented as a Tomcat Servlet using an XML database, and includes an integrated FTP server for managing multimedia content. The server provides support during and post survey. During the survey the server receives and distributes records and GPS locations from each client. If the user drifts out of network coverage the recorded data is cached and flushed to the server when the link is resumed.

\footnotetext{
${ }^{2}$ We used KelvinConnect’s (http://www.kelvinconnect.com) KC form engine
} 
For post-survey services the XML records can be converted into a variety of formats including HTML, allowing the automated creation of a web site which can display categorised coverage maps of various findings, for example to display a colour coded map of the areas where features of a certain age were found.

\section{Evaluation}

The final evaluation of the system took place during a five day archaeological survey in Kirkcudbrightshire, Scotland. The purpose of the survey was to record the GPS location of all known and suspected sites containing features of interest, from the Mesolithic to the Middle Bronze Age. As the survey area was much larger than the wireless range of the equipment, the server was deployed on a laptop acting as a portable access point.
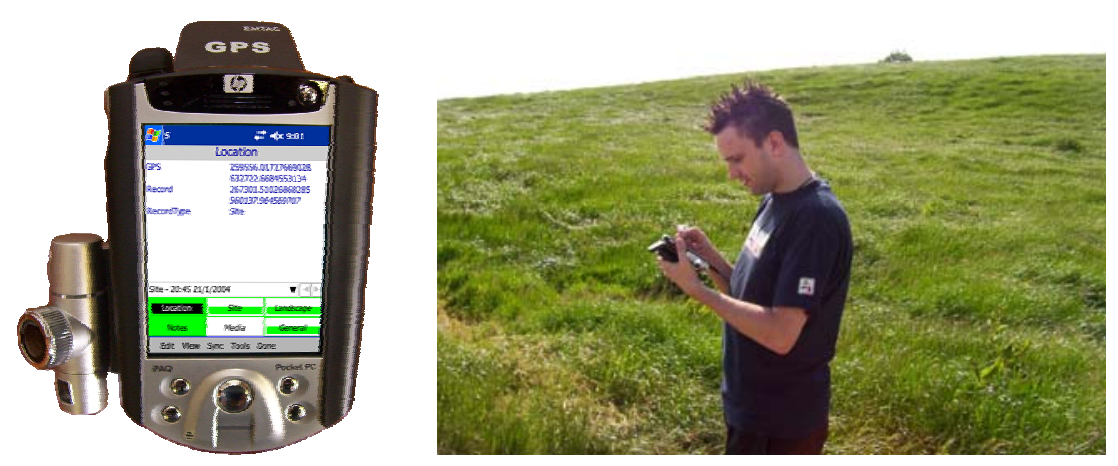

Fig. 2. The system on an iPAQ equipped with camera and GPS

We used four HP iPAQs with integrated 802.11b, GPS and digital camera. Figure 2 displays the client configuration as used in the trials. This compact, lightweight configuration possesses most of the functionality that field workers usually require and can be held comfortably in the hand.

The expedition was successfully completed within the allotted time without any serious problems. For half the expedition time, the participants were asked to use their normal techniques and practices for the survey and for the remaining time to utilise the new facilities provided by our system. During the five days of the trial, over twohundred record entries were made. While the system used was only a prototype it successfully supported collaborative field work. In particular, fieldworkers could easily see which sections of the area had already been surveyed, avoiding the duplicated work we had observed as part of the Melos project.

One problem with the system concerned familiarisation, since many of the surveyors were not experienced with using handheld technology. Another issue discovered was the intangible nature of the data collecting process; users were not completely satisfied with not having hard copies of their reports at hand, even though their observations were cached on their palmtops and readily available at any time. 
However, the most experienced surveyors in the group provided positive comments on the system as it automated many tasks which previously were performed manually. The most useful of these features, identified by the users, was the GPS positioning on the map which reduced the effort required for navigation and automated record location entry.

While the short nature of this trial ruled out collecting extensive data on the effectiveness of the system, we believe that a noticeable increase in productivity would be apparent after prolonged use and familiarisation with the system. In our future work we plan to test this system over a longer period of time to judge its effectiveness with respect to existing paper based technologies.

\section{Conclusion}

We developed a system to facilitate collaboration and coordination in large field surveys. Our approach is unique in focus, realising effective team work whilst replicating the useful functionality of common surveyor tools. To evaluate the system we put it to use in an actual archaeological survey and received positive feedback.

In the future we aim to expand on the systems decentralised nature by implementing part of the server's functionality on the client side. Although a central repository is still useful, by decentralising the system it is possible to exploit the potential of ad hoc networks to greatly expand the coverage area of the system. Finally, it would be interesting to note and observe in practice the potentially wide applicability of such a tool in other scientific domains such as geographical or environmental surveys.

\section{References}

1. Fagrell, H., K. Forsberg, and J. Sanneblad. FieldWise: a mobile knowledge management architecture. in Proceedings of the 2000 ACM conference on Computer supported cooperative work. 2000: ACM Press.

2. Jason Pascoe, N.R., and David Morse, Using while moving: HCI issues in fieldwork environments. ACM Transactions on Computer Human Interaction, 2000. 7(3): p. 417-437.

3. N.S.Ryan, J.P., and D.R.Morse, FieldNote: extending a GIS into the field, in New Techniques for Old Times: Computer Applications in Archaeology, 1998, J.A.Barcelo, I.Briz, and A.Vila, Editors. 1999.

4. Renevier, P. and L. Nigay. Mobile Collaborative Augmented Reality: the Augmented Stroll. in EHCI. 2001. Toronto.

5. Ancona, M., G. Dodero, and V. Gianuzzi. RAMSES: a mobile computing system for field archaeology. in Handheld and Ubiquitous Computing. 1999. Karlsruhe.

6. Gardner, M., et al. Data Capture for Clinical Anaesthesia on a Pen-based PDA: Is It a Viable Alternative to Paper? in HCI. 2001. Lille. 